\title{
ORIGINAL
}

\section{PRESCRIPCIÓN DE VACUNAS NO INCLUIDAS EN EL CALENDARIO VACUNAL EN LA COMUNITAT VALENCIANA DURANTE EL PERÍODO 2004-2009}

\author{
Ana Ruiz Palacio, Eliseo Pastor Villalba, Rosa Martín Ivorra, Ana María Alguacil \\ Ramos, Antonio Portero Alonso y José Antonio Lluch Rodrigo.
}

Servicio de Promoción de la Salud. Dirección General de Salud Pública. Conselleria de Sanitat de la Comunitat Valenciana.

\begin{abstract}
RESUMEN
Fundamentos: En el marco de las políticas de uso racional del medicamento, y al objeto de conseguir una gestión eficiente de los programas de vacunaciones, el objetivo de este trabajo es conocer el número de envases de las vacunas prescritas no incluidas en los programas de vacunación en la Comunitat Valenciana y en sus departamentos de salud, así como el gasto que produjeron en 2009, y analizar la evolución desde 2004, centrando el análisis en la vacuna heptavalente conjugada frente al Streptococcus pneumoniae en menores de dos años.
\end{abstract}

Método: Estudio descriptivo retrospectivo de las vacunas prescritas mediante receta en la Comunitat Valenciana durante el año 2009 y su evolución desde 2004. Variables: número de envases, tipo de beneficiario (activo/pensionista), departamento y gasto generado. Fuentes: Gestor de Prestación Farmacéutica (GAIA) y Sistema Información Poblacional (SIP).

Resultados: En 2009 la prescripción mediante receta de vacunas no incluidas en los programas de vacunación generó un gasto de $683.445,71$ $€$ correspondiente a 17.353 envases, lo que supuso el $87 \%$ del total del gasto en vacunas recetadas. La vacuna frente al $S$. pneumoniae generó el $72 \%$ del gasto total de las vacunas no incluidas en el calendario. La evolución 2004-2009 muestra un aumento del gasto de $735.334 €(24,66 \%)$ en 2005 a partir del cual se produjo un descenso acusado y paulatino que alcanzó los 1.562.650,67 € (-228.64\%). El gasto por departamentos para la vacuna del neumococo conjugada heptavalente por mil niños/as menores de dos años osciló entre 17.377 y $324 €$.

Conclusiones: La tendencia descendente del gasto en recetas prescritas se mantuvo durante 2009 , fundamentalmente de vacunas conjugadas frente a neumococo. No obstante, se observó gran variabilidad interdepartamental en las tasas de prescripción que debe ser corregida.

Palabras clave: Gasto. Prescripción. Vacunas neumococo conjugadas. Utilización de medicamento

\section{$\overline{\text { Ana Ruiz Palacio }}$}

C/Dr. Calatayud Bayá 4-13

46015 Valencia

Correo electrónico: aruizpa@hotmail.com

\section{ABSTRACT}

Public Health Service Prescriptions of Vaccines not Included in Systematic Vaccination Programs in Valencian Community, Spain, during the Period 2004-2009

Background: In the context of the policies of rational use of medicine, and in order to achieve an efficient management of the vaccinations programs, we expect to know the number of packings and cost of prescribed vaccines not included in the vaccination programs of Valencian Community and its departments during 2009 and to analyze its evolution since 2004, focusing on an analysis of Heptavalent pneumococcal conjugate vaccine in children under two years old.

Methods: Retrospective descriptive study to analyze the prescriptions of vaccines in Valencian Community during 2009 and its evolution since 2004. Variables: vaccine availability, number of packings, group of beneficiary (actives/pensioners), department, and cost of prescriptions. Data sources: Gestor de Prestación Farmacéutica (GAIA) and Sistema Información Poblacional (SIP).

Results: In 2009 prescribed vaccines on official national health system prescription forms that are not included in vaccination programs, supposed a cost of $683.445,71 €$ corresponding to 17.353 packings ( $87 \%$ of the total prescribed vaccines). Heptavalent pneumococcal conjugate vaccine generated $72 \%$ of the total cost of vaccines not included in the vaccination programs. The trend from 2004 to 2009 shows an increase in expenditure of $735.334 €(24,66 \%)$ in 2005 from which there takes place a marked and gradual decrease that reaches $1.562 .650,67 €(-228.64 \%)$. The cost by departments of prescriptions per 1000 children under two years old of pneumococcal conjugate vaccine ranges between 17.377 and $324 €$.

Conclusions: The declining trend of prescriptions, mainly of pneumococcal conjugate vaccines, continues during 2009. A great interdepartmental variability is observed, nevertheless, in rates of prescription that should be corrected.

Key words: Drug Costs. Prescription. Heptavalent Pneumococcal conjugate vaccine. Drug utilization. 


\section{INTRODUCCIÓN}

La Ley de Garantías y Uso Racional de los Medicamentos y Productos Sanitarios ${ }^{1}$ considera necesario que la financiación selectiva y no indiscriminada de medicamentos se realice en función de la utilidad terapéutica de los mismos y de su necesidad para mejorar la salud de los ciudadanos. Así mismo, su prescripción y dispensación deberá realizarse de acuerdo con los criterios básicos de uso racional que se establecen en esta Ley. En esta política de uso racional del medicamento deben también incluirse las vacunas.

Como es sabido, el gasto farmacéutico español es, en porcentaje del gasto sanitario, uno de los más altos de Europa, a pesar de la contención experimentada en los últimos años ${ }^{2}$. Sin embargo, el gasto generado por la prescripción de vacunas a través de receta es, en proporción, muy bajo, tanto en nuestra comunidad $(0,41 \%$ del total de gasto farmacéutico) como en las restantes, ya que la mayoría de ellas se encuentran incluidas en los calendarios de vacunaciones.

La provisión de las vacunas de uso sistemático incluidas en los calendarios de vacunación se realiza por las comunidades autónomas mediante compras centralizadas a través de Concursos Públicos, según establece la Ley de Contratos del Sector Público $^{3}$. En el caso de la Comunitat Valenciana, la Dirección General de Salud Pública adquiere anualmente mediante concursos públicos las vacunas que conforman el Calendario de Vacunaciones Sistemático Infantil ${ }^{4}$ y el Programa de Vacunación en Adultos ${ }^{5}$.

Las vacunas incluidas en los calendarios de vacunación son administradas sin coste alguno para la población en los puntos de vacunación públicos y privados de los veintitrés departamentos de salud. Dichos departamentos se corresponden con las diferentes demarcaciones geográficas en las que está dividida la Comunitat Valenciana a efectos sanitarios. Esta ordenación se realiza en función de criterios geográficos, demográficos, socioeconómicos, culturales, epidemiológicos y de dotación de vías y medios de comunicación y las instalaciones sanitarias ${ }^{6}$. Cada departamento de salud tiene asignada una población cuyo rango oscila entre los 50.958 habitantes del departamento 8 y los 373.083 habitantes del departamento 10 .

La prescripción de estas vacunas a través de receta, así como del resto de vacunaciones de uso no sistemático que no son adquiridas por compra centralizada, implica un gasto directo para la Consellerìa de Sanitat que debe ser monitorizado y minimizado de acuerdo con las políticas de uso racional del medicamento.

La vacuna neumocócica conjugada heptavalente se comercializó en Estados Uni$\operatorname{dos}^{7}$ por primera vez en octubre del año 2000 y en Europa $^{8}$ en el año 2001, destacándose, desde entonces, grandes variaciones en sus criterios de utilización en los distintos países europeos ${ }^{9}$. Desde su comercialización en nuestro país es recomendada por muchos pediatras a pesar de no estar incluida en la mayoría de calendarios de vacunación de las comunidades autónomas ni en el del Consejo Interterritorial del Sistema Nacional de Salud, lo que genera situaciones de inequidad, pues no hay razón epidemiológica que justifique las diferencias de su uso entre las diferentes comunidades autónomas. Actualmente solo hay dos comunidades autónomas que la tienen incluida en su calendario, la Comunidad de Madrid desde el año 2006 y Galicia desde enero del 2011. En el resto de comunidades autónomas, la vacuna es de diagnóstico hospitalario y requiere visado de la inspección a efectos de prescripción y dispensación para su financiación por el Sistema Nacional de Salud. De ahí que el Ministerio de Sanidad no 
disponga de datos centralizados por comunidades, lo que implica una dificultad a la hora de poder realizar comparaciones.

La Subdirección General de Asistencia y Prestación Farmacéutica del Ministerio de Sanidad y Consumo restringe el uso de la vacuna a determinados grupos de riesgo $^{10}$ en base a que existe un riesgo más alto de desarrollar una infección neumocócica o de presentar formas graves si existen procesos crónicos o enfermedades de base que alteran la capacidad inmunológica del huésped. Los grupos de riesgo en los que está indicada son:

- Niños inmunocompetentes con riesgo de enfermedad neumocócica o sus complicaciones debido a enfermedades crónicas cardiovasculares o respiratorias, diabetes mellitus o pérdida de líquido cefalorraquídeo.

- Niños inmunocomprometidos con riesgo de enfermedad neumocócica o sus complicaciones debido a asplenia anatómica o funcional, enfermedad de Hodgkin, leucemia, linfoma, insuficiencia renal, síndrome nefrótico, anemia falciforme, trasplante de órganos.

- Niños con infección por VIH, sintomáticos o asintomáticos.

- Personas con trasplante coclear o que van a someterse al mismo.

- Aquellas situaciones que determinen las autoridades sanitarias.

Para estos grupos de riesgo la vacuna sí está incluida en los calendarios de vacunación de la mayoría de comunidades autónomas y se administra de manera gratuita.

Los objetivos de este trabajo son conocer el número de envases de vacunas prescritas mediante receta y el gasto generado en 2009 en los veintitrés departamentos de salud, y analizar la evolución de la prescripción de la vacuna conjugada heptavalente frente al Streptococcus pneumoniae (Prevenar®) y el gasto sanitario que ha implicado desde el año 2004, así como su tasa de prescripción por departamentos de salud.

\section{MATERIAL Y MÉTODOS}

Se analizó la evolución de la prescripción mediante recetas de las vacunas no disponibles en el calendario vacunal sistemático infantil y en los programas de vacunación del adulto.

Se entiende por vacunas disponibles aquellas que están incluidas en el calendario de vacunación sistemático infantil o en el programa de vacunación del adulto y que se suministran desde los almacenes de salud pública. Dentro de estas se encuentran las siguientes: DTPa, Td, Triple Vírica, Hepatitis B pediátrica, Hepatitis B adultos, Hepatitis B hemodializados, Haemophilus influenza tipo B, Hepatitis A pediátrica, Hepatitis A adultos, Pentavalente (Difteria, Tétanos, Pertussis acelular, Haemophilus influenza tipo B, Polio inyectable), Hexavalente (Difteria, Tétanos, Pertussis acelular, Haemophilus influenza tipo B, Polio inyectable y Hepatitis B), Polio inyectable, Meningococo C conjugado, Neumococo 23-valente (polisacáridos), vacuna frente al virus del papiloma humano (VPH), Varicela, Gripe, BCG y Rabia.

Las vacunas no disponibles son aquellas que no están incluidas en el calendario de vacunaciones sistemáticas o en el programa de vacunación del adulto. Dentro de este grupo hemos incluido las vacunas frente a Neumococo 7-valente, Neumococo 10-valente, Tifoidea oral, Tifoidea inyectable, Cólera y Meningococo $\mathrm{A}+\mathrm{C}$.

Las vacunas frente al Neumococo 7valente y Neumococo 10-valente, aunque 
están en los almacenes de los centros de salud pública, no están incluidas en el calendario sistemático de vacunación infantil y se administran sólo a niños con algún factor de riesgo.

El período de estudio fue del 1 de enero del año 2004 al 31 de diciembre del 2009.

Se realizó un análisis descriptivo retrospectivo de las vacunas prescritas mediante receta oficial del Sistema Nacional de Salud durante dicho período. Las vacunas que se incluyeron en el estudio son las adquiridas mediante receta oficial del Sistema Nacional de Salud y registradas en el Sistema de Información Farmacéutica GAIA. Quedaron excluidas del estudio las vacunas que no fueron prescritas en receta oficial del Sistema Nacional de Salud y por tanto no están registradas en GAIA.

El ámbito del análisis fueron los veintitrés departamentos de la Comunitat Valenciana y los datos, previamente validados, se obtuvieron del sistema de información GAIA.

Las variables incluidas en el estudio fueron: disponibilidad de la vacuna (se definen como vacunas disponibles aquellas incluidas en los calendarios de vacunación infantil y del adulto de la Comunitat Valenciana), número de envases, gasto de las recetas prescritas, tipo de beneficiario (activo/pensionista) y departamento de salud.

La fuente de datos utilizada para la obtención de las recetas de vacunas prescritas fue el Sistema de Información Farmacéutica (GAIA), y para el cálculo de los denominadores de población se utilizó el Sistema de Información Poblacional (SIP) de la Conselleria de Sanitat.

GAIA es un sistema central de información de la prestación farmacéutica integrado en el resto de los sistemas existentes en los centros de salud en el entorno (ABUCASIS), y permite a los diferentes agentes que intervienen en el ciclo de prescripción y dispensación disponer de la información necesaria para realizar cada tarea profesional con el máximo de eficiencia, incidiendo en las áreas que, de una u otra forma, constituyen el todo de la prestación farmacéutica.

Se diferencian dos áreas claras de utilidad del sistema GAIA: una de gestión y administración farmacéutica y otra para la prescripción y dispensación.

El Sistema de Información Poblacional (SIP) es una base de datos poblacional creada como registro administrativo que reúne los datos de identificación, localización, acreditación de prestaciones sanitarias y de relación de todas las perosnas valencianas con los servicios de salud.

A partir del Sistema de Información Farmacéutica (GAIA) y del Sistema de Información Poblacional (SIP) de la Consellerìa de Sanitat se obtuvieron los datos de envases prescritos y el gasto generado por cada una de las vacunas no disponibles estudiadas en cada uno de los grupos de beneficiarios en la Comunitat Valenciana en 2009, así como el número de envases prescritos y gasto generado por el total de las vacunas no disponibles estudiadas en cada uno de los departamentos de salud y en cada uno de los grupos de beneficiarios (activos/pensionistas/total).

En base a estos datos se han calculado los correspondientes porcentajes de gasto de las vacunas no disponibles por tipo de beneficiario (sobre el gasto generado por el total de las vacunas estudiadas en cada uno de los grupos de beneficiarios: activos/pensionistas/total), así como de gasto de las vacunas no disponibles por departamento y tipo de beneficiario en la Comunitat Valenciana en 2009 (sobre el gasto generado por el total de las vacunas estu- 
diadas en todos de departamentos de salud de la Comunitat Valenciana por cada uno de los grupos de beneficiarios en ese mismo año). Igualmente se calculó el gasto por mil habitantes de las vacunas no disponibles por departamento de salud de la Comunitat Valenciana por tipo de beneficiario en el año 2009, sobre el número de habitantes en cada uno de los departamentos y en cada uno de los grupos de beneficiarios (activos/pensionistas/total) en ese mismo año.

La evolución del número de envasesgasto de las vacunas no disponibles por tipo de beneficiario durante el periodo 2004-2009 en la Comunitat Valenciana se ha obtenido restando el número de envases-gasto durante los años 2005, 2006, 2007, 2008 y 2009, al número de envasesgasto en 2004. Datos obtenidos igualmente de los sistemas de información anteriormente reseñados, y a partir de los cuales se han calculado los correspondientes porcentajes.

Los indicadores número de envasesgasto por cada mil niños/as menores de dos años mediante recetas de la vacuna conjugada heptavalente frente al $S$. pneumoniae en los departamentos de salud de la Comunitat Valenciana en el año 2009, se han obtenido con datos de las mismas fuentes, e igualmente el indicador evolución del gasto por cada mil niños/as menores de dos años de la vacuna conjugada heptavalente frente al $S$. pneumoniae en el periodo 2005-2009 en los departamentos de la Comunitat Valenciana.

\section{RESULTADOS}

El gasto farmacéutico en vacunas no disponibles prescritas mediante receta en la Comunitat Valenciana durante el año 2009 fue de 17.353 envases, lo que supuso un gasto de $683.445,71 €$ que representó el $87,25 \%$ del total del gasto en vacunas recetadas en el mismo periodo $(783.326,46 €)$.
Del total de envases prescritos en el año 2009, $15.904(91,64 \%)$ se prescribieron en población infantil, siendo sus padres beneficiarios activos.

La tabla 1 expresa cómo la vacuna más prescrita durante el año 2009 fue la vacuna oral frente a la fiebre tifoidea con $7.791(44,90 \%)$ envases y un gasto de $62.639,64 €$, lo que supuso el $9,17 \%$ del gasto total. Sin embargo, la que mayor peso tuvo sobre el gasto farmacéutico fue la vacuna heptavalente conjugada frente al neumococo, con 6.558 envases que supusieron el $37,79 \%$ respecto al total de los envases prescitos de vacunas y un gasto de $491.584,156 €$, que correspondió al $71,93 \%$ del total de gasto respecto al resto de vacunas prescritas en el año 2009.

Tal como refleja la tabla 2, el mayor volumen de prescripción de vacunas no disponibles mediante recetas corresponde al departamento 10 con 2.810 envases y un gasto de $152.905,55 €$, seguido del departamento 23 con 1.107 envases y un gasto de 68.376,35 € y el departamento 9 con 2.028 envases y un gasto de $52.607,55 €$, representando entre los tres el $40 \%$ del total del gasto en el año 2009. En la figura 1 se expresa el gasto medio por cada mil habitantes y departamento de salud durante el año 2009, observándose como el 21 y el 22 presentaron los valores inferiores, con menos de $25 €$ de gasto por mil habitantes, frente a los más de $400 €$ de los departamentos 10 y 23 .

La tabla 3 refleja la evolución de la prescripción mediante receta de las vacunas no disponibles desde el año 2004 hasta el 2009. Destaca el año 2005 con 61.916 envases prescritos y un gasto de $2.981 .430,28 €$. A partir de entonces y hasta el 2009 se produjo un descenso gradual tanto en el número de envases como en el gasto. 


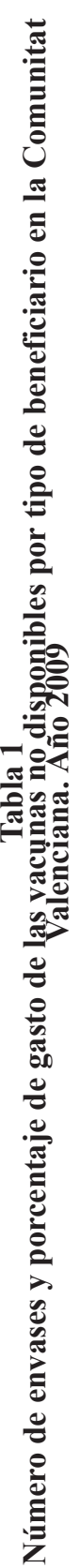

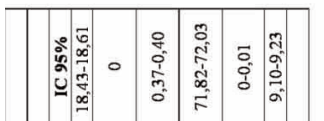

象:

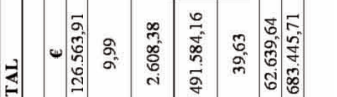

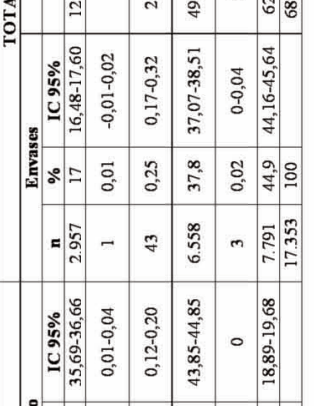

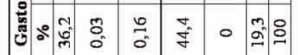

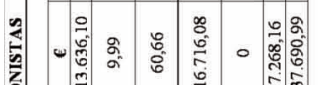

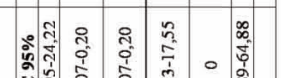

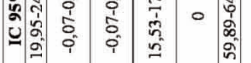

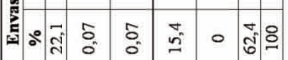

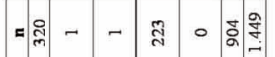

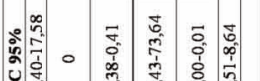

2n $n$ n

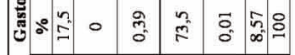

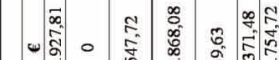

औी

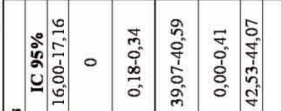

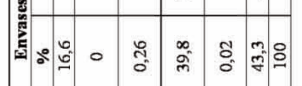

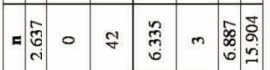

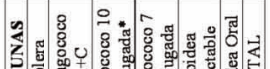

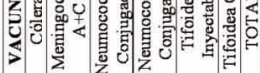

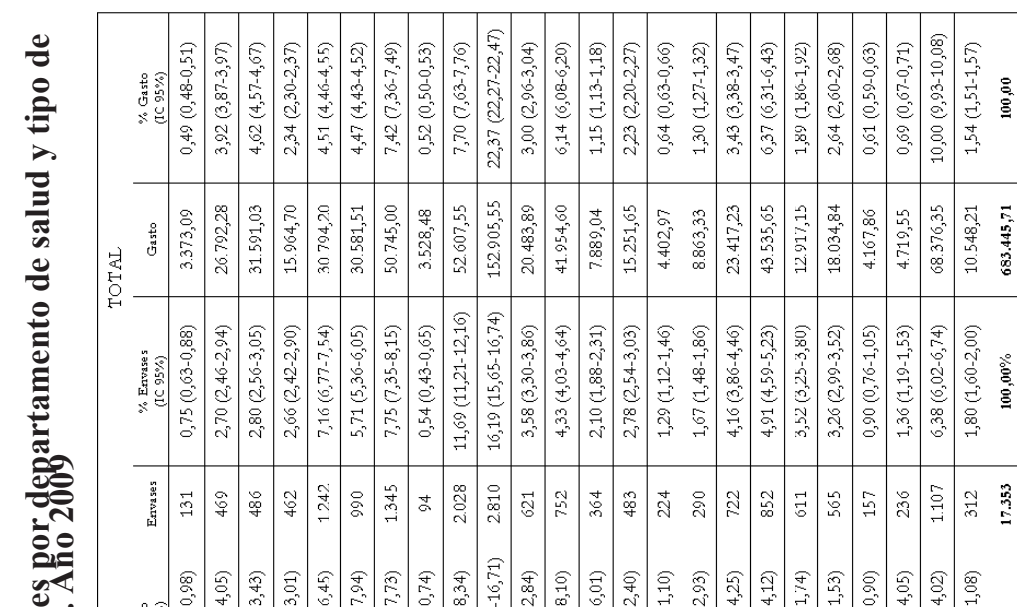

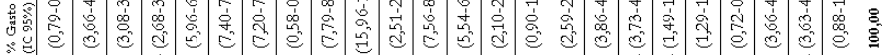

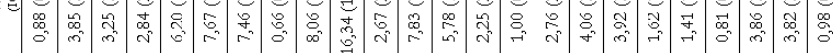

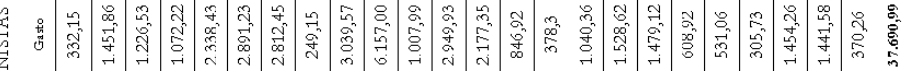

N

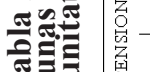

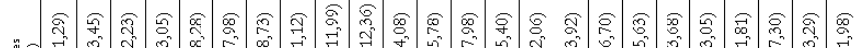

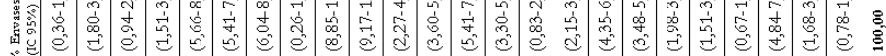

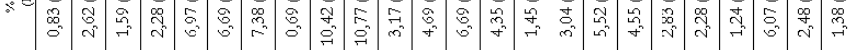

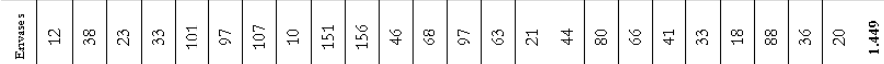

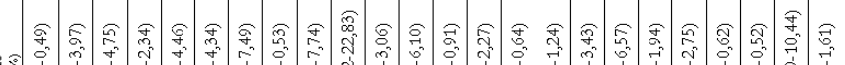

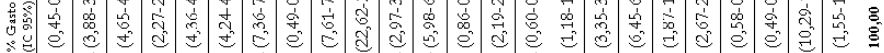

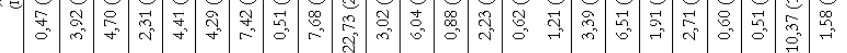

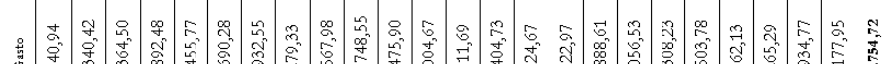

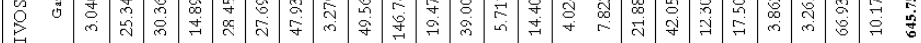
若

包

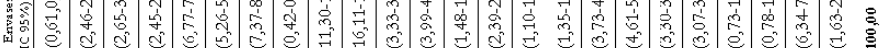

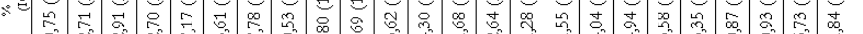

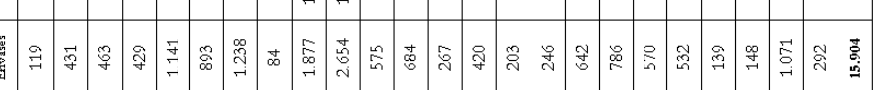




\section{Figura 1}

Gasto por mil habitantes de las vacunas no disponibles por departamento de salud de la Comunitat Valenciana, por tipo de beneficiario. Año 2009

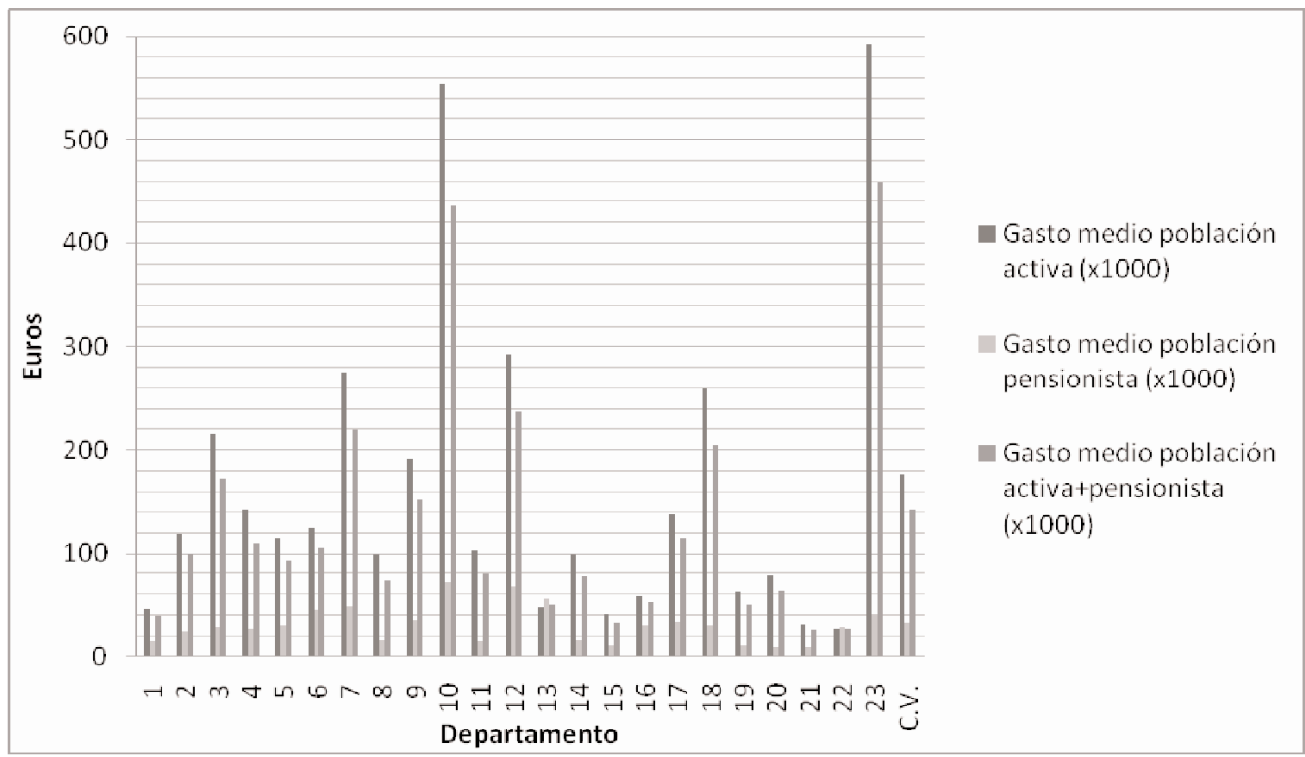

Fuentes: SIP y GAIA. No se han tenido en cuenta los habitantes sin derecho a farmacia

Tabla 3

Evolución del número de envases y gasto de las vacunas no disponibles por tipo de beneficiario durante el periodo 2004-2009 en la Comunitat Valenciana

\begin{tabular}{|c|c|c|c|c|c|c|c|c|c|c|}
\hline & \multicolumn{3}{|c|}{2004} & \multicolumn{3}{|c|}{2005} & \multicolumn{3}{|c|}{2006} \\
\hline & & $\mathbf{n}$ & $\%$ & IC 95\% & $\mathbf{n}$ & $\%$ & IC $95 \%$ & $\mathbf{n}$ & $\%$ & IC $95 \%$ \\
\hline \multirow{5}{*}{ cavases } & A & 35.644 & 89,77 & $89,47-90,07$ & 49.573 & 80,06 & $79,75-80,38$ & 47.024 & 82,62 & $82,31-82,94$ \\
\hline & $\mathbf{P}$ & 4.063 & 10,23 & $9,93-10,53$ & 12.343 & 19,94 & $19,62-20,25$ & 9.889 & 17,38 & $17,06-17,69$ \\
\hline & $\mathrm{T}$ & 39.707 & 100 & & 61.916 & 100 & & 56.913 & 100 & \\
\hline & $\mathbf{E}$ & & & & 22.209 & 35,87 & & 17.206 & 30,20 & \\
\hline & & $\boldsymbol{\epsilon}$ & $\%$ & IC $95 \%$ & $\boldsymbol{\epsilon}$ & $\%$ & IC $95 \%$ & $\boldsymbol{\epsilon}$ & $\%$ & IC 95\% \\
\hline \multirow{4}{*}{ gasto } & A & $2.157,436,97$ & 96,05 & $96,03-96,08$ & $2.809 .632,06$ & 94,24 & $94,21-94,26$ & $2.589,837,35$ & 94,58 & $94,55-94,61$ \\
\hline & $\mathbf{P}$ & $88.659,41$ & 3,95 & $3,92-3,97$ & $171.798,22$ & 5,76 & $5,74-5,79$ & $148.419,97$ & 5,42 & $5,39-5,45$ \\
\hline & $\mathrm{T}$ & $2.246 .096,38$ & 100 & & $2.981 .430,28$ & 100 & & $2.738 .257,32$ & 100 & \\
\hline & $\mathbf{E}$ & & & & $735.333,90$ & 24,66 & & 492160.94 & 18,00 & \\
\hline & & \multicolumn{3}{|c|}{2007} & \multicolumn{3}{|c|}{2008} & \multicolumn{3}{|c|}{2009} \\
\hline & & $\mathbf{n}$ & $\%$ & IC $95 \%$ & $\mathbf{n}$ & $\%$ & IC $95 \%$ & n & $\%$ & IC $95 \%$ \\
\hline \multirow{5}{*}{ envases } & A & 41.844 & 84,32 & $84,00-84,64$ & 28.427 & 82,20 & $81,81-82,61$ & 15.904 & 91,65 & $91,24-92,06$ \\
\hline & $\mathbf{p}$ & 7.783 & 15,68 & $15,36-16,00$ & 6.152 & 17,80 & $17,39-18,19$ & 1.449 & 8,35 & $7,94-8,76$ \\
\hline & $\mathrm{T}$ & 49.627 & 100 & & 34.579 & 100 & & 17.353 & 100 & \\
\hline & E & 9.920 & 20 & & -5.128 & $-14,80$ & & -22.354 & -128 & \\
\hline & & $\boldsymbol{\epsilon}$ & $\%$ & IC 95\% & $\boldsymbol{\epsilon}$ & $\%$ & IC 95\% & E & $\%$ & IC 95\% \\
\hline \multirow{4}{*}{ gasto } & A & $2.152 .023,61$ & 94,55 & $94,52-94,58$ & $1.232 .514,62$ & 92,60 & $92,60-92,69$ & $645.754,72$ & 94,48 & $94,43-94,54$ \\
\hline & $\mathbf{P}$ & $124.134,89$ & 5,45 & $5,42-5,48$ & $97.892,77$ & 7,40 & $7,31-7,40$ & $37.690,99$ & 5,52 & $5,46-5,57$ \\
\hline & $\mathrm{T}$ & $2.276 .158,50$ & 100 & & $1.330 .407,39$ & 100 & & $683.445,71$ & 100 & \\
\hline & E & $30.062,12$ & 1,32 & & $-915.688,99$ & $-68,83$ & & $-1.562 .650,67$ & $-228,64$ & \\
\hline
\end{tabular}

A: Activos, P: Pensionistas, T: Total, E: Evolución respecto al año 2004. 


\section{Tabla 4}

Tasa de número de envases y gasto por cada mil niños/as menores de dos años mediante recetas de la vacuna conjugada heptavalente frente al $S$. pneumoniae en los departamentos de salud de la Comunitat Valenciana. Año 2009

\begin{tabular}{|c|c|c|c|c|c|c|c|c|c|c|}
\hline \multirow{2}{*}{$\begin{array}{c}\text { Dptos } \\
1\end{array}$} & \multirow{2}{*}{$\begin{array}{c}\text { nủmero } \\
\text { anvases }\end{array}$} & \multirow{2}{*}{$\begin{array}{c}\text { \% envases } \\
0,41 \%\end{array}$} & \multicolumn{2}{|c|}{ IC 95\% } & \multirow{2}{*}{$\begin{array}{l}\text { gasto }(\Theta) \\
2023,92\end{array}$} & \multirow{2}{*}{$\begin{array}{l}\text { \% gasto } \\
0,41 \%\end{array}$} & \multicolumn{2}{|c|}{ IC 95\% } & \multirow{2}{*}{$\begin{array}{c}\text { anvases } x \\
1000 \\
\text { niffos/as }< \\
2 \text { anfos } \\
14\end{array}$} & \multirow{2}{*}{$\begin{array}{c}\begin{array}{c}\text { gasto } X \\
1000 \\
\text { niflos/as }\end{array} \\
<2 \text { aftos } \\
1.032 .09 \\
\end{array}$} \\
\hline & & & $0,26 \%$ & $0.57 \%$ & & & $0.39 \%$ & $0,43 \%$ & & \\
\hline 2 & 298 & $4,54 \%$ & $4,04 \%$ & $5,05 \%$ & $22.338,08$ & $4,54 \%$ & $4,49 \%$ & $4,60 \%$ & 46 & $3.478,37$ \\
\hline 3 & 380 & $5,79 \%$ & $5,23 \%$ & $6,36 \%$ & $28.484,80$ & $5,79 \%$ & $5,73 \%$ & $5,86 \%$ & 83 & $6.188,31$ \\
\hline 4 & 140 & $2,13 \%$ & $1,78 \%$ & $2,48 \%$ & $10.494,40$ & $2,13 \%$ & $2.09 \%$ & $2,18 \%$ & 41 & $3.052,47$ \\
\hline 5 & 95 & $1,45 \%$ & $1,16 \%$ & $1,74 \%$ & $7.121,20$ & $1,45 \%$ & $1,42 \%$ & $1,48 \%$ & 12 & 932,7 \\
\hline 6 & 233 & $3.55 \%$ & $3,10 \%$ & $4.00 \%$ & $17,465,68$ & $3,55 \%$ & $3,50 \%$ & $3,60 \%$ & 31 & 2313,95 \\
\hline 7 & 468 & $7,14 \%$ & $6,51 \%$ & $7,76 \%$ & $35.081,28$ & $7,14 \%$ & $7,06 \%$ & $7,21 \%$ & 92 & $6.880,03$ \\
\hline 8 & 31 & $0,47 \%$ & $0,31 \%$ & $0,64 \%$ & 2323,76 & $0,47 \%$ & $0,45 \%$ & $0,49 \%$ & 34 & 2528.57 \\
\hline 9 & 273 & $4,16 \%$ & $3,68 \%$ & $4,65 \%$ & $20.464,08$ & $4,16 \%$ & $4,11 \%$ & $4,22 \%$ & 34 & 2576,04 \\
\hline 10 & 1.786 & $27,23 \%$ & $26,1 \oplus \%$ & $28,31 \%$ & $133.876,80$ & $27,23 \%$ & $27,11 \%$ & $27,36 \%$ & 221 & $16.550,48$ \\
\hline 11 & 180 & $2,74 \%$ & $2,35 \%$ & $3,14 \%$ & $13.492,80$ & $2,74 \%$ & $2,70 \%$ & $2,79 \%$ & 31 & 2301,74 \\
\hline 12 & 491 & $7,49 \%$ & $6,85 \%$ & $8,12 \%$ & $36.805,36$ & $7,49 \%$ & $7,41 \%$ & $7,56 \%$ & 121 & $9.085,50$ \\
\hline 13 & 39 & $0,59 \%$ & $0,41 \%$ & $0,78 \%$ & 2923,44 & $0,59 \%$ & $0,57 \%$ & $0,62 \%$ & 12 & 935,2 \\
\hline 14 & 133 & $2,03 \%$ & $1,69 \%$ & $2,37 \%$ & $9.969,68$ & $2,03 \%$ & $1,99 \%$ & $2,07 \%$ & 32 & 2372,60 \\
\hline 15 & 15 & $0,23 \%$ & $0,11 \%$ & $0,34 \%$ & $1.124,40$ & $0,23 \%$ & $0,22 \%$ & $0,24 \%$ & 5 & 394,39 \\
\hline 16 & 71 & $1,08 \%$ & $0,83 \%$ & $1,33 \%$ & $5,322,16$ & $1,08 \%$ & $1,05 \%$ & $1,11 \%$ & 20 & 1.502 .59 \\
\hline 17 & 211 & $3,22 \%$ & $2,79 \%$ & $3,64 \%$ & $15.814,80$ & $3,22 \%$ & $3,17 \%$ & $3,27 \%$ & 45 & $3,341,39$ \\
\hline 18 & 505 & $7,70 \%$ & $7,06 \%$ & $8,35 \%$ & $37.854,80$ & $7,70 \%$ & $7,63 \%$ & $7,78 \%$ & 106 & $7.951,02$ \\
\hline 19 & 65 & $0,999 \%$ & $0,75 \%$ & $1,23 \%$ & $4.872,40$ & $0,99 \%$ & $0,96 \%$ & $1,02 \%$ & 11 & 802,44 \\
\hline 20 & 148 & $2,26 \%$ & $1,90 \%$ & $2,62 \%$ & $11.094,08$ & $2.26 \%$ & $2,22 \%$ & $2,30 \%$ & 21 & $1.600,42$ \\
\hline 21 & 26 & $0,40 \%$ & $0,24 \%$ & $0,55 \%$ & $1.948,96$ & $0,40 \%$ & $0,38 \%$ & $0,41 \%$ & 6 & 456,32 \\
\hline 22 & 15 & $0,23 \%$ & $0,11 \%$ & $0,34 \%$ & $1.124,40$ & $0,23 \%$ & $0,22 \%$ & $0,24 \%$ & 4 & 324,78 \\
\hline 23 & 851 & $12.98 \%$ & $12,1 \%$ & $13,79 \%$ & $63,790,96$ & $12,98 \%$ & $12,88 \%$ & $13,07 \%$ & 232 & $17,377,00$ \\
\hline Otros" & 77 & $1,17 \%$ & $0,91 \%$ & $1,43 \%$ & $5.771,92$ & $1,17 \%$ & $1,14 \%$ & $1,20 \%$ & S.D. & S.D. \\
\hline C.V. & 6.558 & 100,00 & 100,00 & 100,00 & $491.584,16$ & 100,00 & 100,00 & 100,00 & 59 & $4.420,96$ \\
\hline
\end{tabular}

Incluye las recetas prescritas por médicos no adscritos a ningún departamento como numerador y como denominador la población no adscrita a ningún departamento de salud.

\section{Figura 2}

Evolución del gasto por cada mil niños/as menores de dos años de la vacuna conjugada heptavalente frente al $S$. pneumoniae en el periodo 2005-2009 en los departamentos de salud de la Comunitat Valenciana

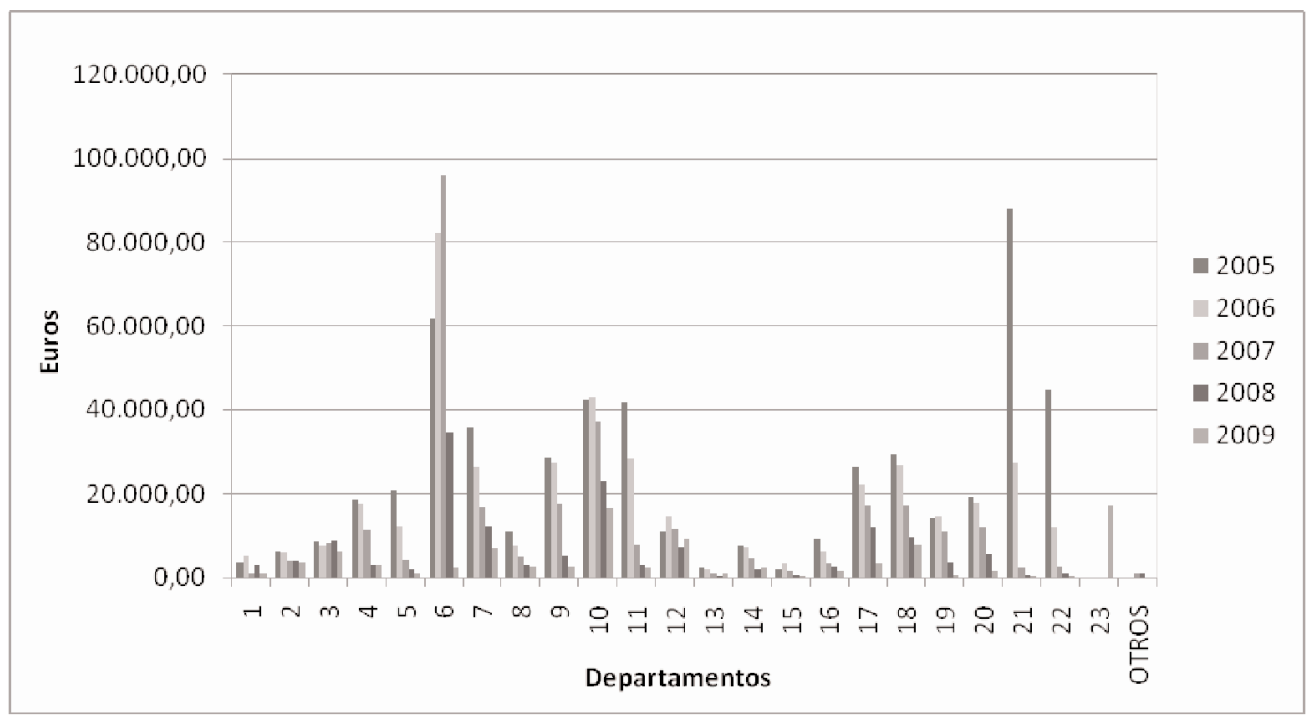

Otros: Incluye las recetas prescritas por médicos no adscritos a ningún departamento 
La tabla 4 recoge la tasa de envases prescritos y de gasto por departamento por cada mil niños/as menores de dos años correspondientes a la vacuna conjugada heptavalente frente al $S$. pneumoniae, quedando reflejado un amplio rango que osciló entre los 232 envases del departamento 23 y $\operatorname{los} 4$ del 22.

Tal como expresa la figura 2 , se produjo un descenso anual para el período de estudio del gasto generado por la vacuna de $S$. pneumoniae prescrita mediante receta por cada mil niños/as menores de dos años.

\section{DISCUSIÓN}

La fiebre tifoidea es una enfermedad cuya incidencia en Europa ha descendido de forma brusca, siendo la tasa de incidencia por 100.000 habitantes de 0,21 $\operatorname{casos}^{11}$, presentándose en forma de casos aislados y no habiéndose publicado brotes en España desde el año 2000. Así pues, desde el punto de vista epidemiológico, no sería una vacuna que debiera prescribirse con tanta frecuencia como se refleja en el análisis de los datos (salvo en el caso de viajeros).

La vacuna frente al neumococo 7-valente, desde su comercialización en España, y a pesar de haberse acordado que su uso fuera restringido a los grupos de riesgos citados anteriormente, ha suscitado polémica a la hora de la prescripción tanto entre los profesionales sanitarios como entre la población. Este hecho ha supuesto que los pediatras no hayan mantenido una postura clara a la hora de prescribir la vacuna, lo que explica la variabilidad del número de envases recetados entre los diferentes departamentos.

Se observa una tendencia descendente del gasto generado por las vacunas prescritas no incluidas en el calendario de vacunaciones sistemáticas infantiles y en el programa del adulto en la Comunitat
Valenciana a partir del año 2005 , en contraposición al gasto farmacéutico global de nuestra comunidad que ha aumentado aunque, si bien es verdad, en una menor proporción año a año ${ }^{12}$. A ello han podido contribuir dos acciones implantadas desde la Conselleria de Sanitat, que la solicitud de la vacuna frente a neumococo (su precio es el que más influye en el gasto total) por parte de los pediatras a los almacenes de los Centros de Salud Pública deba estar debidamente justificada (para ello han de cumplimentar un impreso con su nombre y el motivo por el que se prescribe) y la elaboración de un informe de gestión que ha de remitirse a las gerencias de los diferentes departamentos de salud para que lo difundan entre los coordinadores de los centros de salud y los pediatras de los mismos.

Es por ello que, aun no habiéndose medido el impacto de ambas medidas, sí se puede concluir (tomando como dato objetivo de la prescripción el número de envases prescritos), que éstas han dado como resultado una gestión más eficiente por parte de los profesionales sanitarios, pediatras sobre todo, en la prescripción de vacuna frente al neumococo 7 -valente.

La variabilidad existente entre departamentos en relación a la prescripción de la vacuna frente al $S$. pneumoniae significa que no en todos se ha seguido la instrucción de prescribir la vacuna únicamente a los niños menores de dos años que presentaban algún factor de riesgo, tal como se informó a los pediatras y direcciones de Atención Primaria de cada uno de los veintitrés departamentos de salud. La variabilidad entre los distintos departamentos hace necesario que se siga insistiendo en la homogeneización de los criterios a la hora de prescribir la vacuna, sobre todo en los que presentan tasas más elevadas, pues epidemiológicamente no se aprecia un patrón diferente en los factores de riesgo de los niños de los departamen- 
tos con mayores tasas de prescripción que aconsejen la indicación de la vacuna conjugada heptavalente frente a $S$. pneumoniae. Este esfuerzo debe ser exigido no solo a los prescriptores sino también a otras estructuras de gestión, como es el caso de la inspección de farmacia, cuyo visado es necesario para la adquisición mediante receta de esta vacuna.

Con el fin de que la eficiencia de la prescripción sea cada vez mayor y se minimice la variabilidad entre los diferentes profesionales sanitarios es necesario que, además de dar instrucciones claras desde la Administración Sanitaria sobre la prescripción frente a la vacuna neumocócica heptavalente, se establezca un feed-back con los médicos prescriptores cuando se observe que su indicación es superior a la media de la Comunitat Valenciana, no dándose criterios epidemiológicos que justifiquen la vacunación de forma universal.

Junto con la vacuna contra el neumococo, son las del cólera y la fiebre tifoidea oral, en segundo y tercer lugar respectivamente, las que han implicado un coste más elevado entre las vacunas no incluidas en los calendarios de vacunación. Éstas se recomiendan ante situaciones muy específicas, generalmente relacionadas con viajes internacionales a países del tercer mundo cuya frecuencia está aumentando en los últimos años. La vacunación frente a la fiebre tifoidea se recomienda a viajeros a países considerados endémicos, especialmente a quienes realicen estancias largas y a las personas expuestas a condiciones de higiene deficitarias. Estos criterios deberían ser conocidos por los facultativos de modo que se evite la disparidad o improcedencia en el momento de la prescripción.

En relación con las limitaciones de este trabajo cabe destacar, por un lado, la inexistencia de otros estudios de gasto sobre prescripción de vacunas no inclui- das en los programas de vacunaciones oficiales, lo cual ha impedido la realización de un análisis comparativo. Por otro, resaltar que los trabajos publicados sobre gasto farmacéutico hacen referencia a la Dosis Diaria Definida por mil habitantes. Esta unidad de medida es independiente del precio y de la formulación de los medicamentos, lo que permite evaluar la evolución del consumo de los mismos y realizar comparaciones entre grupos de población $\mathrm{y}$ entre sistemas de atención de salud. No obstante, en el caso de las vacunas (y en el de los medicamentos tópicos, anestésicos generales o locales, medios de contraste y extractos de alérgenos) no se puede calcular la Dosis Diaria Definida por mil habitantes, pues las dosis prescritas varían en función de la edad de comienzo de la vacunación, de las dosis previas recibidas y de las circunstancias individuales del vacunado (viajes, riesgo por existir una patología de base, etc.).

Por último, convendría valorar la probabilidad de que en los dos últimos años del estudio, variables como la crisis económica hayan podido influir en que en los grupos de niños sin riesgo y familias con un poder adquisitivo bajo se hayan administrado menos dosis de vacuna 7 -valente o 10 -valente.

\section{AGRADECIMIENTOS}

A José Luis Trillo Mata, Jefe de Área de la Dirección General de Farmacia y Productos Sanitarios, Noelia Selles Carpena y Rocío Broseta Solaz de la oficina de Gestión de Abucasis de la Agencia Valenciana de la Salud.

\section{BIBLIOGRAFÍA}

1. Boletín Oficial del Estado. Ley 29/2006 de 26 de Julio de garantías y uso racional de los medicamentos y productos sanitarios. BOE núm.178 de 27/07/2006.

2. Sánchez Bayle M. Control del gasto farmacéutico: un avance, aunque insuficiente. ElPaís. 2010 
Marzo 29. Periódico digital [Citado 1 de junio 2010]. Disponible en:

www.elpais.com/articulo/sociedad/Control/gasto/farmaceutico/avance/insuficiente/elpepusoc/20100329elpepusoc_6/Tes.

3. Boletín Oficial del Estado. Ley 34/2010 de 5 de agosto de modificación de diversas Leyes, entre otras la Ley 30/2007, de 30 de octubre, de Contratos del Sector Público, BOE núm.192 de 09/08/2010.

4. Diario Oficial de la Comunitat Valenciana. Orden de 16 de Julio de 2008 Vacunación Sistemática Infantil en la Comunidad Valenciana. DOCV núm. 5812 de 23/07/2008.

5. Diario Oficial de la Comunitat Valenciana. Orden de 13 de Abril de 2005 Programa de Vacunación en Adultos en la Comunidad Valenciana. DOCV núm. 5016 de 30/05/2005.

6. Diario Oficial de la Comunitat Valenciana. Decreto $74 / 2007$, de 18 de mayo, del Consell, por el que se aprueba el Reglamento sobre estructura, organización y funcionamiento de la atención sanitaria en la Comunitat Valenciana. DOCV núm. 5518 de $23 / 05 / 2007$.

7. Pneumococcal 7-valent Conjugate Vaccine (Diphtheria CRM197 Protein) Prevnar. Package insert. Wyeth Pharmaceuticals Inc. Philadelphia, PA. Version dated october 2008. Disponible en: http://www.fda.gov/BiologicsBloodVaccines/Vaccines/ApprovedProducts/ucm180017.html. Citado el $18 / 02 / 2011$

8.European Medicines Agency (EMEA). European Public Assessment Report (EPAR) for Prevenar. Revision 14. London: EMEA; 2009 Jan 27. Disponible en:

http://www.emea.europea.eu/humandocs/Humans/E $\mathrm{PAR} /$ prevenar/prevenar.htm

9. De Carvalho Gomes H, Muscat M, Monnet DL, Giesecke J, Lopalco PL. Use of seven-valent pneumococcal conjugate vaccine (PCV7) in Europe., 2001-2007. EuroSurveillance 2009; 14(12). Disponible en: http://www.eurosurveillance.org/ViewArticle.aspx?ArticleId=19159

10. Resolución de 22 de junio de 2001 de la Dirección General de Farmacia y Productos Sanitarios. Ministerio de Sanidad y Consumo.

11. Hernández Cabrera M, Pérez Arellano JL. Fiebre Tifoidea en Manual de Vacunaciones en Pediatría. Comité Asesor de Vacunas $4^{\mathrm{a}}$ edición. Bilbao. Asociación Española de Pediatría; 2008.p. 425-38.
12.El gasto farmacéutico se cerró en 2009 con un crecimiento del 4,47\%. El Farmacéutico. 2010 Enero 27. Revista electrónica [Citado 1 de junio $2010] . \quad$ Disponible en : www.elfarmaceutico.es/index.php/actualidad/788-el-gasto-farmaceutico-se-cerro-en-2009con-un-crecimiento-del-447.html 